\title{
Variable Refocusing Flip Angle Single-Shot Imaging for Sedation-Free Fast Brain MRI
}

\author{
(D) R. Jabarkheel, (D)E. Tong, (D)E.H. Lee, (D)T.M. Cullen, (D). Yousaf, (D)A.M. Loening, (D). Taviani, (D) Michael Iv., (D) G.A. Grant,
} (D) S.J. Holdsworth, DS.S. Vasanawala, and (D) K.W. Yeom

\begin{abstract}
BACKGROUND AND PURPOSE: Conventional single-shot FSE commonly used for fast MRI may be suboptimal for brain evaluation due to poor image contrast, SNR, or image blurring. We investigated the clinical performance of variable refocusing flip angle single-shot FSE, a variation of single-shot FSE with lower radiofrequency energy deposition and potentially faster acquisition time, as an alternative approach to fast brain MR imaging.
\end{abstract}

MATERIALS AND METHODS: We retrospectively compared half-Fourier single-shot FSE with half- and full-Fourier variable refocusing flip angle single-shot FSE in 30 children. Three readers reviewed images for motion artifacts, image sharpness at the brain-fluid interface, and image sharpness/tissue contrast at gray-white differentiation on a modified 5-point Likert scale. Two readers also evaluated full-Fourier variable refocusing flip angle single-shot FSE against T2-FSE for brain lesion detectability in 38 children.

RESULTS: Variable refocusing flip angle single-shot FSE sequences showed more motion artifacts $(P<.001)$. Variable refocusing flip angle single-shot FSE sequences scored higher regarding image sharpness at brain-fluid interfaces $(P<.001)$ and gray-white differentiation $(P<.001)$. Acquisition times for half- and full-Fourier variable refocusing flip angle single-shot FSE were faster than for single-shot FSE $(P<.001)$ with a $53 \%$ and $47 \%$ reduction, respectively. Intermodality agreement between full-Fourier variable refocusing flip angle single-shot FSE and T2-FSE findings was near-perfect $(\kappa=0.90, \kappa=0.95)$, with an $8 \%$ discordance rate for ground truth lesion detection.

CONCLUSIONS: Variable refocusing flip angle single-shot FSE achieved $2 \times$ faster scan times than single-shot FSE with improved image sharpness at brain-fluid interfaces and gray-white differentiation. Such improvements are likely attributed to a combination of improved contrast, spatial resolution, SNR, and reduced T2-decay associated with blurring. While variable refocusing flip angle single-shot FSE may be a useful alternative to single-shot FSE and, potentially, T2-FSE when faster scan times are desired, motion artifacts were more common in variable refocusing flip angle single-shot FSE, and, thus, they remain an important consideration before clinical implementation.

ABBREVIATIONS: SSFSE $=$ single-shot FSE; vrfSSFSE $=$ variable refocusing single-shot FSE

F ast MR imaging sequences commonly used to image infants and young children are often a derivative of T2-weighted MR imaging with shorter acquisition times. ${ }^{1}$ While fast MR imaging sequences have, to date, shown lower image quality compared with standard T2-weighted FSE MR imaging, they do provide a faster method for evaluating the global brain and are particularly useful for assessing fluid-filled spaces or structures. ${ }^{2}$ Historically,

Received December 24, 2019; accepted after revision April 18, 2020. From the Stanford University School of Medicine (R.J.); and Departments of Radiology (E.T., A.M.L., V.T., M.I.), Electrical Engineering (E.H.L.), and Neurosurgery (G.A.G.), Stanford University, Stanford, California; Department of Radiology (T.M.C., U.Y., S.S.V., K.W.Y.), Lucile Packard Children's Hospital, Stanford University, Palo Alto, California; and Department of Anatomy and Medical Imaging and Centre for Brain Research (S.J.H.), Faculty of Medical and Health Sciences, University of Auckland, Auckland, New Zealand.

This work was supported by National Institutes of Health grants R01 EB009690, P41 EB015891, 1R21HD08380301A1; the Tashia and John Morgridge Faculty Scholars fund; GE Healthcare; and the ASNR Comparative Effective Research Award. fast MR imaging was clinically implemented to reduce the radiation exposure of serial CT imaging in shunted children and avoid sedation often required for lengthier conventional MR imaging scans on children. ${ }^{3-5}$

There are various types of fast MR imaging, including singleshot FSE (SSFSE), balanced steady-state free precession, and hybrid methods using a combination of gradient- and spin-echoes. ${ }^{2,6-11}$

Paper previously presented, in part, at: International Pediatric Radiology Conjoint Meeting and Exhibition, May 15-20, 2016; Chicago, Illinois.

Please address correspondence to Kristen W. Yeom, MD, Department of Radiology, Stanford University, 725 Welch Rd MC 5654, Stanford, CA, 94304; e-mail: kyeom@stanford.edu; @RJabarkheel; @KristenYeom

\footnotetext{
- Indicates open access to non-subscribers at www.ajnr.org

$\equiv$ Indicates article with supplemental on-line tables.

Indicates article with supplemental on-line photos.

http://dx.doi.org/10.3174/ajnr.A6616
} 
All of these techniques are fast and therefore easy to implement without sedation. However, either limited resolution and blurring (SSFSE), poor tissue contrast (balanced steady-state free precession), or sensitivity to off-resonance and system imperfections (gradient- and spin-echoes) limits these fast MR imaging sequences from being used for broader clinical applications beyond evaluation of fluid structures. The PROPELLER method may combat motion and provide higher resolution. ${ }^{12}$ PROPELLER is an FSE-based method, whereby several parallel data lines that form "blades" are acquired in a radial (or propeller-like) $k$-space acquisition. Because the blades rotate around the center of the $k$-space, this central oversampling provides redundancy of information, which can be used for motion correction. Nonetheless, PROPELLER poses challenges of lengthy acquisition times, requiring a greater degree of patient cooperation.

Among the various fast MR imaging approaches, SSFSE has emerged as one of the most frequently used pulse sequences, largely because of its T2-weighted contrast, speed, and robustness to motion, off-resonance, and system imperfections. ${ }^{13}$ However, SSFSE has limitations due to its high rate of radiofrequency energy deposition-particularly at $3 \mathrm{~T}$-which translates into high specific absorption rate values. ${ }^{14}$ Delays between the acquisition of consecutive slices need be introduced, resulting in section TRs longer than the time necessary to simply play the pulse sequence waveforms, to remain within the FDA specific absorption rate guidelines. While this issue is more pronounced when using the body coil for excitation, a delay is still required when using a transmit/receive head coil. ${ }^{15}$

Recently, a variation of SSFSE with variable refocusing flip angles (variable refocusing single-shot FSE [vrfSSFSE]) has been described. ${ }^{16}$ This flip angle modulation was originally introduced to reduce T2-decay-associated blurring, mostly in the context of volumetric imaging. ${ }^{17-19}$ Coincidentally, the flip angle modulation also reduces radiofrequency energy deposition and thus the specific absorption rate; hence, the aforementioned delays between acquisitions of successive slices can be shortened, resulting in faster imaging. Despite the potential benefits of faster imaging, the image quality of the brain using low refocusing flip angle SSFSE remains unknown. Furthermore, with vrfSSFSE, signal levels tend to oscillate over the echo train and are, thus, more vulnerable to motion-related signal loss, which might impede clinical interpretability. In this study, we sought to investigate a faster MR imaging approach that incorporates vrfSSFSE and examine its potential clinical applicability. ${ }^{20}$

\section{MATERIALS AND METHODS}

\section{Subjects Undergoing SSFSE and vrfSSFSE}

Our institution, the Lucile Packard Children's Hospital (Palo Alto, California) has integrated vrfSSFSE as part of our fast MR imaging brain protocol since April 2014. After institutional review board approval with waived consent, we retrospectively identified 30 consecutive children referred for noncontrast, sedation-free fast brain MR imaging between December 2015 and April 2016. Children who had undergone back-to-back conventional half-Fourier SSFSE, half-Fourier vrfSSFSE, and full-Fourier vrfSSFSE sequences at $3 \mathrm{~T}$ MR imaging were included. The median age of patients was 6 years (age range, 8 months to 20 years).
Clinical characteristics of patients who had SSFSE and vrfSSFSE images are listed in On-line Table 1.

\section{Subjects Undergoing T2-FSE and Full-Fourier vrfSSFSE}

We also retrospectively identified 38 consecutive children who had undergone back-to-back T2-FSE and full-Fourier vrfSSFSE scans at 3T MR imaging in October 2016 as part of a qualityassurance project. The median age of patients who had both T2FSE and full-Fourier vrfSSFSE scans was 6 years (age range, 1 week to 17 years). Clinical characteristics of patients who had T2-FSE and full-Fourier vrfSSFSE images are listed in On-line Table 2.

\section{Imaging Methods}

All subjects were scanned on a 3T Discovery MR750 scanner (GE Healthcare) with an 8-channel head coil.

SSFSE and vrfSSFSE Image Acquisition. The MR imaging protocol comprised 3 orthogonal planes of SSFSE (constant refocusing flip angle of $130^{\circ}$ ) and coronal vrfSSFSE. SSFSE parameters were the following: FOV $=18-22 \mathrm{~cm}$ (adjusted to each patient's anatomy), array coil spatial sensitivity encoding parallel imaging factor $=2$, half-Fourier with homodyne reconstruction, effective $\mathrm{TE}=86 \mathrm{~ms}$, matrix $=256 \times 256$, and bandwidth $=83 \mathrm{kHz}$. Contiguous, 4-mm-thick slices with interleaved section ordering were used. The vrfSSFSE sequences had the same parameters, but auto-calibrating parallel imaging was used instead of array coil spatial sensitivity encoding. Full-Fourier vrfSSFSE was performed in addition to half-Fourier vrfSSFSE with homodyne reconstruction. ${ }^{21}$ The TR for each sequence was recorded.

T2-FSE and Full-Fourier vrfSSFSE Image Acquisition. Axial fullFourier vrfSSFSE parameters were the following: section thickness $=4 \mathrm{~mm}, 0.5$ skip, FOV $=18-22 \mathrm{~cm}$, matrix $=256 \times 256$, average TR/TE $=960 / 160 \mathrm{~ms}$. Axial T2-FSE autocalibrating reconstruction for Cartesian imaging parameters were the following: section thickness $=4 \mathrm{~mm}, 0.5$ skip, FOV $=18-22 \mathrm{~cm}$, matrix $=$ $512 \times 256$, with average TR/TE times $=3000 / 100 \mathrm{~ms}$.

\section{SSFSE versus vrfSSFSE Image-Quality Evaluation}

Two blinded, board-certified neuroradiologists with Certificates of Added Qualification (K.W.Y., $>10$ years; M.I., $>5$ years' experience) and 1 pediatric radiology fellow (T.M.C.) independently evaluated coronal half-Fourier SSFSE, half-Fourier vrfSSFSE, and full-Fourier vrfSSFSE sequences in random order with regard to individual patients and the sequence type. The reviewers assessed the following image features: motion artifacts, image sharpness at the brain-fluid interfaces (eg, cortical surface, cerebral sulci, or cerebellar fissures), and image sharpness within the brain substance, specifically targeting tissue contrast at the gray-white differentiation. Motion artifacts were scored on the basis of motion-related signal drop-out. Sharpness took into account factors that contributed to better visualization of boundaries of solid-fluid and solidsolid structures. Note that higher sharpness scores could be attributed to a combination of factors, including improved image contrast (such as contrast at the cortical surface or within the brain parenchyma at the gray-white differentiation), improved spatial resolution and SNR, and reduced T2-decay-associated blurring. For 


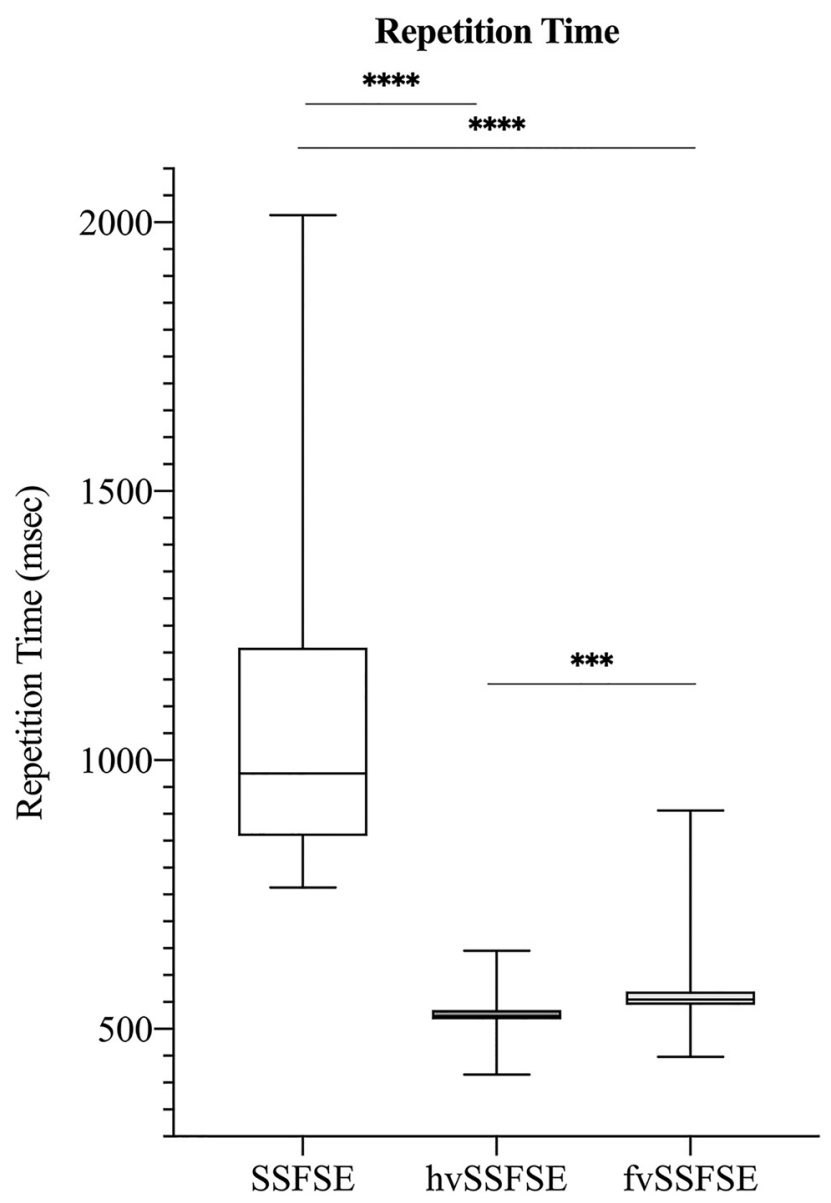

FIG 1. TRs of SSFSE and vrfSSFSE. Box-and-whisker plots for each sequence are shown with the middle bar representing the median, the box representing the 25th and 75th percentiles, and the upper and lower bars representing the range. Half-Fourier vrfSSFSE (hvSSFSE) and full-Fourier vrfSSFSE (fvSSFSE) have significantly faster TRs compared with SSFSE $(P<.001)$. hvSSFSE has a significantly faster TR compared with fvSSFSE $(P<.001)$. All comparisons of TRs were assessed using the Mann-Whitney test.

scoring, we used the following modified 5-point Likert scale: 1, nondiagnostic; 2 , limited study; 3 , suboptimal quality; 4 , not affecting diagnostic quality, minimal artifacts or image-quality problems; 5 , diagnostic, no artifacts or excellent image quality.

Evaluation of T2-FSE and Full-Fourier vrfSSFSE Image Findings Two blinded board-certified neuroradiologists with Certificates of Added Qualification (K.W.Y. and E.T., >2 years' experience) independently reviewed axial T2-FSE and full Fourier vrfSSFSE images in a random order and with at least a 2-week interval to avoid recall bias between the 2 sequences. The reviewers recorded all detectable pathologic brain lesions. T2-FSE served as the ground truth. The full-Fourier vrfSSFSE findings were considered in agreement with T2-FSE only if all abnormal findings found on T2-FSE were also detected on full-Fourier vrfSSFSE in an all-or-none grading manner. We did not evaluate agreement for minor T2*-related features, such as focal hemosiderin or mineralization.

\section{Statistical Analysis}

Wilcoxon matched-pairs signed ranked tests were used to compare radiologists' ratings between SSFSE and vrfSSFSE sequences.
Mann-Whitney tests were used to compare TRs between SSFSE and vrfSSFSE sequences. Interrater agreement for SSFSE and vrfSSFSE image-quality evaluation was assessed with the Fleiss $\kappa$ statistic because we had 3 readers. Following interrater agreement analyses between SSFSE and vrfSSFSE raw scores, Fisher exact tests were used to compare the fraction of scans rated highly, 4 or 5 , for each image-quality parameter for SSFSE and vrfSSFSE sequences. Intermodality agreement between full-Fourier vrfSSFSE and T2FSE findings for a given reader was assessed using the Cohen $\kappa$. Wilcoxon matched-pairs signed rank tests, Mann-Whitney tests, Fisher exact tests, and Cohen $\kappa$ were all calculated using GraphPad Prism software (Version 8.2.1; GraphPad Software). The Fleiss $\kappa$ statistic was calculated using Excel (Version 16.29; Microsoft).

\section{RESULTS}

Overall, vrfSSFSE sequences showed significantly faster acquisition times compared with SSFSE (Fig 1). Specifically, half-Fourier vrfSSFSE (mean, $528 \mathrm{~ms}$ ) and full-Fourier vrfSSFSE (mean, 597 $\mathrm{ms}$ ) had a $53 \%$ and $47 \%$ decrease in TR, respectively, compared with SSFSE (mean, $1120 \mathrm{~ms} ; P<.001$ ). There was no instance in which either vrfSSFSE or SSFSE was considered nondiagnostic within the narrow clinical scope for which fast MR imaging was obtained (evaluation of ventricular size, cyst, or large fluid collection).

\section{Motion Artifacts}

SSFSE scored significantly higher (ie, had less motion-related signal loss) than both half-Fourier vrfSSFSE and full-Fourier vrfSSFSE (mean, 4.6 versus 3.8 versus 3.7 , respectively; $P<.001$; Fig $2 A$ and Table 1). Figure 3 shows an example of motionrelated signal loss that might be seen with vrfSSFSE. While these artifacts were less common for SSFSE, $77 \%$ and $80 \%$ of halfFourier vrfSSFSE and full-Fourier vrfSSFSE, respectively, showed minimal-to-no artifacts (Table 2).

\section{Image Sharpness at the Brain-Fluid Interfaces}

Both half-Fourier vrfSSFSE and full-Fourier vrfSSFSE scored higher than SSFSE (mean, 4.2 versus 4.1 versus 3.4, respectively; $P<.001$; Fig $2 B$ ). Figure 4 shows an example of improved sharpness with vrfSSFSE, particularly at the cortical-sulcal interface.

\section{Image Sharpness/Tissue Contrast at the Gray-White Differentiation}

Both half-Fourier vrfSSFSE and full-Fourier vrfSSFSE scored higher than SSFSE (mean, 4.4 versus 4.4 versus 3.5, respectively; $P<.001$; Fig $2 C$ ). Figure 5 shows an example of improved image sharpness stemming from higher tissue contrast and spatial resolution and SNR with more distinct gray-white differentiation on vrfSSFSE, including improved anatomic detail of smaller structures such as the cerebellar folia.

\section{Comparison of the vrfSSFSE Sequences}

There were no statistically significant differences for motion artifacts or overall image sharpness.

\section{SSFSE and vrfSSFSE Image-Quality Interrater Agreement}

Raters varied on the basis of the image-quality parameter assessed. There was substantial interrater agreement for motion artifacts 


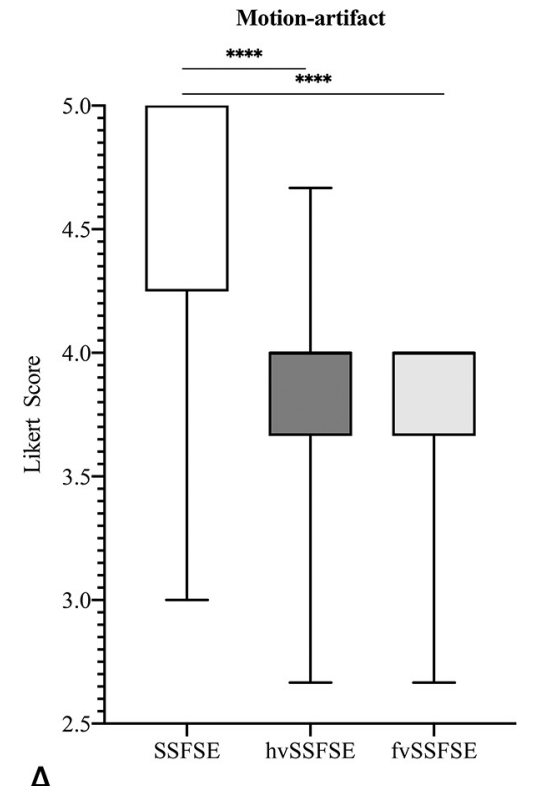

A

FIG 2. Image-quality ratings of SSFSE and vrfSSFSE. Box-and-whisker plots for each image-quality parameter (Motion artifact, Sharpness: brain-fluid and Sharpness: gray-white contrast rated on a 5-point Likert scale) for a given sequence with the middle bar representing the median, the box representing the 25th and 75th percentile, and the upper and lower bars representing the range. A, SSFSE scans scored significantly higher than both hvSSFSE and fvSSFSE scans on motion artifacts $(P<.001)$. hvSSFSE and fvSSFSE scans did not differ significantly for Motion artifact $(P>.5)$. $B$, hvSSFSE and fvSSFSE scans were rated significantly better than SSFSE scans for Sharpness: brain-fluid $(P<.001)$. hvSSFSE and fvSSFSE scans did not differ significantly for Sharpness: brain-fluid $(P>.2)$. C, hvSSFSE and fvSSFSE scans were rated significantly better than SSFSE scans for Sharpness: gray-white contrast $(P<.001)$. hvSSFSE and fvSSFSE scans did not differ significantly for Sharpness: gray-white contrast $(P>$.9). All comparisons of image-quality ratings were made using the Wilcoxon matched-pairs signed-rank test.

Table 1: Mean image-quality ratings of SSFSE and vrfSSFSE scans and interrater agreement ${ }^{a}$

\begin{tabular}{llll}
\hline & Motion Artifact $^{\mathrm{b}}$ & Sharpness: Brain-Fluid $^{\mathbf{c}}$ & Sharpness: gray-white contrast $^{\mathbf{d}}$ \\
\hline SSFSE & $4.6 \pm 0.6$ & $3.4 \pm 0.7$ & $3.5 \pm 0.6$ \\
& $(0.63$, substantial) & $(0.43$, moderate) & $(-0.03$, poor $)$ \\
Half-Fourier vrfSSFSE & $3.8 \pm 0.5$ & $4.2 \pm 0.6$ & $4.4 \pm 0.7$ \\
& $(0.39$, fair) & $(0.32$, fair $)$ & $(0.07$, slight $)$ \\
Full-Fourier vrfSSFSE & $3.7 \pm 0.5$ & $4.1 \pm 0.5$ & $4.4 \pm 0.6$ \\
& $(0.37$, fair) & $(0.23$, fair) & $(0.03$, slight $)$ \\
Pooled & $(0.62$, substantial) & $(0.47$, moderate $)$ & $(0.15$, slight) \\
\hline
\end{tabular}

${ }^{a}$ Wilcoxon matched-pairs signed-rank test was used to compare reader image-quality ratings of sequences.

bSSFSE was scored significantly higher than both half- and full-Fourier vrfSSFSE for Motion Artifact $(P<.001)$.

${ }^{C}$ Both vrfSSFSE sequences were rated significantly higher than SSFSE for Sharpness: Brain-Fluid $(P<.001)$.

${ }^{d}$ Both vrfSSFSE sequences were rated significantly higher than SSFSE for Sharpness: gray-white contrast $(P<.001)$.

$(\kappa=0.62)$. There was moderate agreement for image sharpness at the brain-fluid interfaces $(\kappa=0.47)$ and slight agreement for image sharpness/tissue contrast at gray-white differentiation $(\kappa=0.15)$. Table 1 provides interrater agreement on a pooled-sequence basis for image-quality parameters and on an individual-sequence basis by image-quality parameters. While there is slight-to-moderate interrater agreement for image sharpness at brain-fluid interfaces and gray-white differentiation, both half-Fourier vrfSSFSE and fullFourier vrfSSFSE had significantly more images rated either a 4 (very good) or 5 (excellent) compared with SSFSE for these imagequality parameters $(P<.001$; Table 2$)$.

\section{Comparison of T2-FSE and Full-Fourier vrfSSFSE}

Intermodality agreement between T2-FSE and full-Fourier vrfSSFSE findings for a given reader was almost perfect, with $\kappa=$ 0.90 and $\kappa=0.95$, respectively. Side-by-side examples that compare T2-FSE and full-Fourier vrfSSFSE are shown in Fig 6 and
On-line Fig 1. With T2-FSE as ground truth, full-Fourier vrfSSFSE demonstrated an $8 \%$ discordance rate. One reviewer missed a small cyst or cavity on full-Fourier vrfSSFSE that was detectable on T2-FSE (On-line Fig 2). Both reviewers missed a focal intraparenchymal hemorrhage on full-Fourier vrfSSFSE that was detected on T2-FSE.

\section{DISCUSSION}

Fast MR imaging of the brain has transformed the management of children with shunted hydrocephalus or ventriculomegaly by reducing the radiation exposure of serial CT head examinations and sedation that might be required for a lengthier conventional MR imaging of the brain. Since its initial use for ventricular assessment, however, many pediatric hospitals have increasingly adopted various fast MR imaging methods for other clinical conditions such as cyst evaluation, venous thrombosis, congenital anomalies, Chiari 

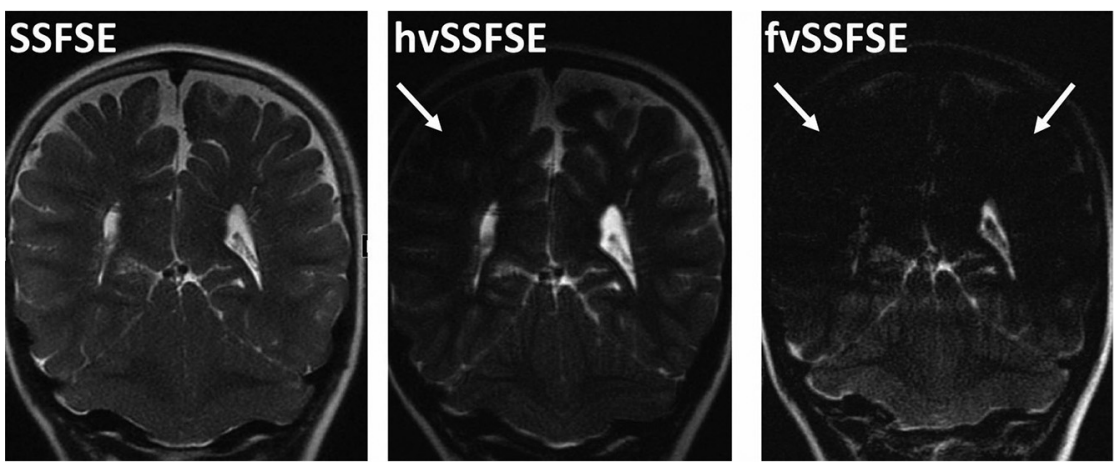

FIG 3. Example of motion-related signal loss. An 8-year-old girl presented for ventricular assessment. Half-Fourier vrfSSFSE (hvSSFSE) and full-Fourier vrfSSFSE (fvSSFSE) images show motionrelated signal loss (arrows) on some of the slices that compromised image detail.

Table 2: Fraction of SSFSE and vrfSSFSE scans rated highly, 4 or 5 , for readers' assessments ${ }^{a}$

\begin{tabular}{lccc}
\hline & $\begin{array}{c}\text { Motion } \\
\text { Artifact }^{\mathrm{b}}\end{array}$ & $\begin{array}{c}\text { Sharpness: Brain- } \\
\text { Fluid }^{\mathbf{c}}\end{array}$ & $\begin{array}{c}\text { Sharpness: gray-white } \\
\text { contrast }^{\text {d }}\end{array}$ \\
\hline SSFSE & $94 \%$ & $37 \%$ & $50 \%$ \\
Half-Fourier vrfSSFSE & $80 \%$ & $91 \%$ & $90 \%$ \\
Full-Fourier vrfSSFSE & $77 \%$ & $96 \%$ & $94 \%$ \\
\hline
\end{tabular}

${ }^{a}$ Fisher exact tests were used to compare the fraction of scans rated highly, 4 or 5 , for each image-quality parameter for SSFSE and vrfSSFSE sequences.

${ }^{b}$ There was no statistically significant difference in the fraction of SSFSE scans rated either a 4 (very good) or 5 (excellent) for Motion-Artifact as compared to vrfSSFSE sequences $(P>.1)$.

${ }^{c}$ Both vrfSSFSE sequences had a higher fraction of images rated either 4 or 5 as compared to SSFSE for Sharpness: Brain-Fluid $(P<.001)$.

${ }^{\mathrm{d}}$ Both vrfSSFSE sequences had a higher fraction of images rated either 4 or 5 as compared to SSFSE for Sharpness: Gray-White Contrast $(P<.0001)$.
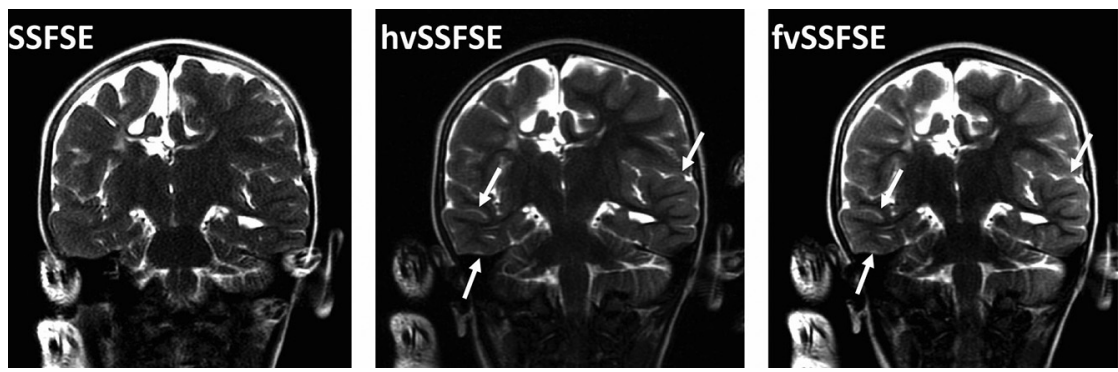

FIG 4. Example of image sharpness or blurring. A 3-year-old girl presented for ventricular assessment. Half-Fourier vrfSSFSE (hvSSFSE) and full-Fourier vrfSSFSE (fvSSFSE) images (arrows) show improved sharpness or reduced blurring, compared with the corresponding conventional SSFSE, particularly along the cortical margins where the cortical surface is better distinguished against the overlying subarachnoid spaces.
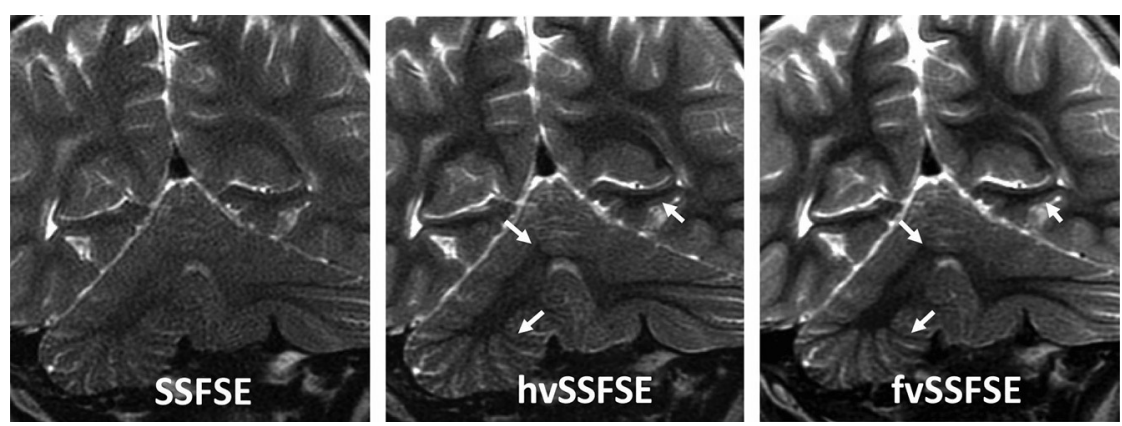

FIG 5. Example of magnified views demonstrating differences in tissue contrast. A 2-year-old boy presented for ventricular assessment. Half-Fourier vrfSSFSE (hvSSFSE) and full-Fourier vrfSSFSE (fVSSFSE) images show improved tissue contrast (arrows) that allows improved visualization of the gray-white junction, cerebellar folia detail, as well as posterior hippocampal regions. malformations, and postoperative hemorrhage. $^{5,11,22}$ Greater recognition of radiation-related cancer risks of repeat head CT examinations, in part, has driven such expanded clinical indications because lifetime cancer mortality risk due to radiation exposure from head CT in a 1-year-old child is estimated to be $0.07 \%$, an order of magnitude higher than that of adults. ${ }^{13,23-25}$ Potential risks and adverse effects of sedation in children are other contributing factors. ${ }^{26}$

Among the various fast MR imaging approaches, SSFSE is frequently used for its speed and excellent T2-weighted contrast. A central limitation of SSFSE, however, is its high rate of radiofrequency energy deposition due to refocusing pulse trains with a constant flip angle, which results in high specific absorption rates. The high specific absorption rate of SSFSE mandates a longer TR, in which motion between acquisitions of sections could lead to misregistration. In this study, we tested whether a variation of SSFSE with variable refocusing flip angles (thus decreasing radiofrequency energy deposition) could reduce TRs and thereby facilitate faster imaging compared with conventional SSFSE. We also sought to compare image quality between vrfSSFSE and conventional SSFSE and then compare the diagnostic performance of vrfSSFSE against T2-FSE used in standard-of-care brain MR imaging protocols.

In this study, we applied variable refocusing flip angles for potential fast MR imaging optimization of the pediatric brain. We found that vrfSSFSE sequences significantly reduced TRs and therefore overall scan times by around 50\%. Although vrfSSFSE was more vulnerable to motion-related signal loss, it gave rise to images with improved tissue contrast, spatial resolution, and SNR, compared with SSFSE and suggested a potential clinical role for vrfSSFSE in evaluating the brain beyond cysts or ventricles.

To address the potential for vrfSSFSE to have an expanded clinical role, we evaluated the clinical performance of vrfSSFSE for evaluating the pediatric brain, including normal 

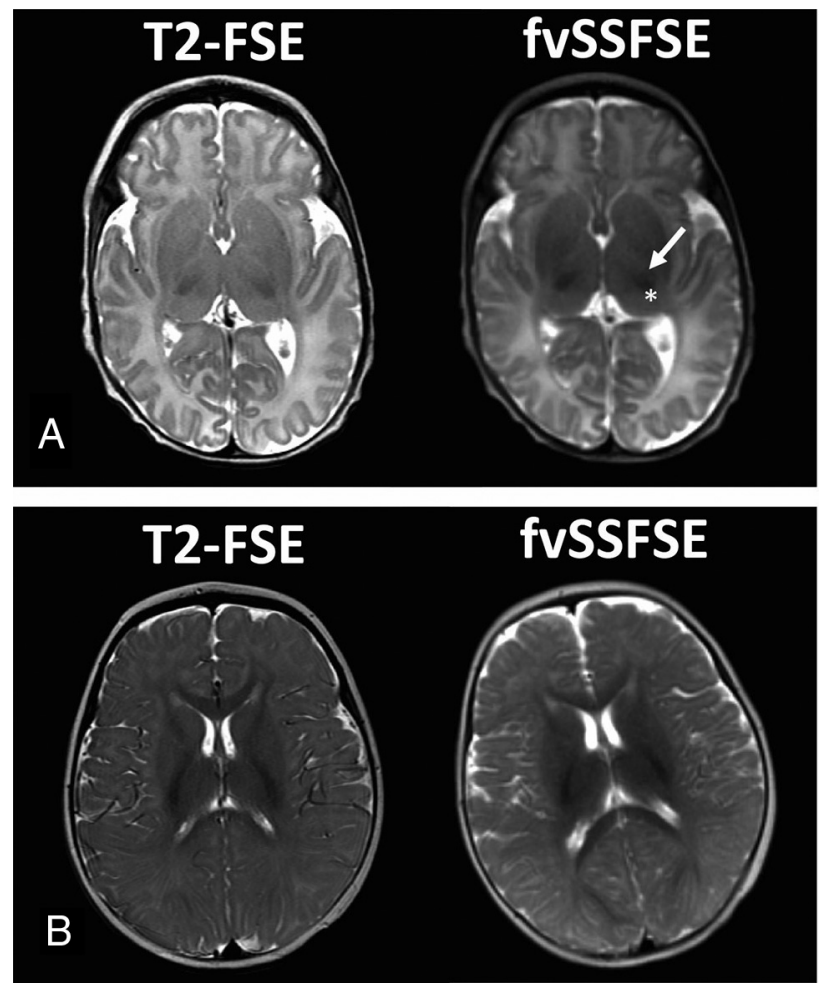

FIG 6. Normal pediatric infant brain. High tissue contrast that is feasible with full-Fourier vrfSSFSE (fvSSFSE) compared with conventional SSFSE robustly delineates both gray-white differentiation as well as the myelination pattern that nearly resembles that of conventional T2-FSE. Because SSFSE is acquired with long echo trains (during which the signal intensity decays), image blurring occurs. This effect arises from the different signal intensities of each echo, depending on their individual TEs. A, An infant with premature birth history, now nearterm in age, presents for screening MR imaging. Note age-appropriate myelination with dark signal in the thalamus (asterisk) and posterior limb of internal capsule (arrow). B, A 6-month-old male infant with spasmus nutans, who presented for screening MR imaging to exclude optic chiasm glioma. In this older infant, myelination has further progressed and is age-appropriate.

and abnormal findings such as brain malformations and injury, etc., across all ages, using T2-FSE as the ground truth. On the basis of our results, vrfSSFSE performed comparable with standardof-care T2-FSE and with near-perfect intermodality agreement, suggesting a potential role for integrating vrfSSFE as an alternative to T2-FSE if anesthesia-free MR imaging or reduced scan times are desired. We also propose that vrfSSFSE could be a faster alternative to PROPELLER, as shown in On-line Fig 3.

A prior study has shown that improved image quality and soft-tissue contrast are feasible by applying a refocusing flip angle optimization framework to the HASTE sequence, generating image contrast on a par with T2-FSE for adult brain tumors. ${ }^{27}$ An important contributor to vrfSSFSE performance in lesion detection might be its image sharpness related to improved tissue contrast and spatial resolution, facilitating lesion detection both at the cortical surface and within the brain substance. Because a variable refocusing flip angle train modifies the signal decay of tissues with different $\mathrm{T} 2$ relaxation constants, an image contrast is produced that is different from the traditional constant flip angle refocusing train. This may potentially make vrfSSFSE better suited to highlighting differences in brain tissue structures, including gray-white differentiation. While vrfSSFSE did miss a few cases of focal hemorrhage, small $\mathrm{T} 2^{*}$-susceptibility lesions may be difficult to detect, even on T2-FSE.

One important limitation was the relative lack of motion in our patient cohort. With low-refocusing flip angle SSFSE, signal tends to oscillate over the echo train and is potentially more vulnerable to motion-related signal loss. ${ }^{20} \mathrm{We}$, therefore, likely underestimated the potential detrimental effects of motion-related signal loss that could impede clinical interpretation. However, there is also a tradeoff in which faster vrfSSFSE might be more successful at quickly imaging motion-prone patients during a quiescent period in the scanner, eg, imaging an infant with a swaddle-and-feed before waking up. Other limitations include suboptimal interrater agreement, particularly for image sharpness/tissue contrast at the gray-white junction-which likely stemmed from subjective differences for what constitutes scores of 4 or 5 . When we examined a subset of scans rated either 4 or 5 for each sequence for a given image quality, we continued to find that vrfSSFSE sequences provided a significant improvement in image sharpness and contrast. It is also possible that there was unconscious bias. While all readers were blinded to sequence types, which were reviewed in random order, it is conceivable that differences between SSFSE and vrfSSFSE sequences outside of image quality affected reported ratings. Another important limitation is our small sample size; a larger cohort study would be needed to more fully assess the clinical utility of low-refocusing flip angle SSFSE for specific neurologic diseases.

\section{CONCLUSIONS}

This study shows that ultra-fast T2 imaging was feasible with variable refocusing flip angles for SSFSE. Although motionrelated signal loss was more prevalent compared with conventional SSFSE, vrfSSFSE came with improved tissue contrast and spatial resolution and SNR. Although the study is limited by the small sample size, our pilot results comparing vrfSSFSE with T2-FSE suggest a potentially broader clinical role for vrfSSFSE, beyond cyst and ventricle evaluation, particularly if sedationfree or a faster MR imaging protocol is considered or when conventional methods are less desirable due to time constraints.

Disclosures: Valentina Taviani-UNRELATED: Employment: GE Healthcare. Shreyas S. Vasanawala—RELATED: Grant: GE Healthcare*; UNRELATED: Consultancy: Arterys, HeartvVista. *Money paid to the institution.

\section{REFERENCES}

1. Iskandar BJ, Sansone JM, Medow J, et al. The use of quick-brain magnetic resonance imaging in the evaluation of shunt-treated hydrocephalus. J Neurosurg 2004;101:147-51 CrossRef Medline

2. Ashley WW Jr, McKinstry RC, Leonard JR, et al. Use of rapidsequence magnetic resonance imaging for evaluation of hydrocephalus in children. J Neurosurg 2005;103:124-30 CrossRef Medline

3. Ahn SS, Mantello MT, Jones KM, et al. Rapid MR imaging of the pediatric brain using the fast spin-echo technique. AJNR Am J Neuroradiol 1992;13:1169-77 Medline

4. Haacke EM, Tkach JA. Fast MR imaging: techniques and clinical applications. AJR Am J Roentgenol 1990;155:951-64 CrossRef Medline

5. Tekes A, Senglaub SS, Ahn ES, et al. Ultrafast brain MRI can be used for indications beyond shunted hydrocephalus in pediatric patients. AJNR Am J Neuroradiol 2018;39:1515-18 CrossRef Medline 
6. Patel MR, Klufas RA, Alberico RA, et al. Half-Fourier acquisition single-shot turbo spin-echo (HASTE) MR: comparison with fast spin-echo MR in diseases of the brain. AJNR Am J Neuroradiol 1997;18:1635-40 Medline

7. Sugahara T, Korogi Y, Hirai T, et al. Comparison of HASTE and segmented-HASTE sequences with a T2-weighted fast spin-echo sequence in the screening evaluation of the brain. AJR Am J Roentgenol 1997;169:1401-10 CrossRef Medline

8. Fellner F, Schmitt R, Trenkler J, et al. Turbo gradient-spin-echo (GRASE): first clinical experiences with a fast T2-weighted sequence in MRI of the brain. Eur J Radiol 1995;19:171-76 CrossRef Medline

9. Rockwell DT, Melhem ER, Bhatia RG. GRASE (gradient- and spinecho) MR of the brain. AJNR Am J Neuroradiol 1997;18:1923-28 Medline

10. Miller JH, Walkiewicz T, Towbin RB, et al. Improved delineation of ventricular shunt catheters using fast steady-state gradient recalledecho sequences in a rapid brain MR imaging protocol in nonsedated pediatric patients. AJNR Am J Neuroradiol 2010;31:430-35 CrossRef Medline

11. Rozovsky K, Ventureyra EC, Miller E. Fast-brain MRI in children is quick, without sedation, and radiation-free, but beware of limitations. J Clin Neurosci 2013;20:400-05 CrossRef Medline

12. Vertinsky AT, Rubesova E, Krasnokutsky MV, et al. Performance of PROPELLER relative to standard FSE T2-weighted imaging in pediatric brain MRI. Pediatr Radiol 2009;39:1038-47 CrossRef Medline

13. Patel DM, Tubbs RS, Pate G, et al. Fast-sequence MRI studies for surveillance imaging in pediatric hydrocephalus. J Neurosurg Pediatr 2014;13:440-47 CrossRef Medline

14. Allison J, Yanasak N. What MRI sequences produce the highest specific absorption rate (SAR), and is there something we should be doing to reduce the SAR during standard examinations? AJR Am J Roentgenol 2015;205:W140 CrossRef Medline

15. Loening AM, Litwiller DV, Saranathan M, et al. Increased speed and image quality for pelvic single-shot fast spin-echo imaging with variable refocusing flip angles and full-Fourier acquisition. Radiology 2017;282:561-68 CrossRef Medline

16. Loening AM, Saranathan M, Ruangwattanapaisarn N, et al. Increased speed and image quality in single-shot fast spin echo imaging via variable refocusing flip angles. J Magn Reson Imaging 2015;42:174758 CrossRef Medline
17. Busse RF. Reduced RF power without blurring: correcting for modulation of refocusing flip angle in FSE sequences. Magn Reson Med 2004;51:1031-37 CrossRef Medline

18. Busse RF, Brau AC, Vu A, et al. Effects of refocusing flip angle modulation and view ordering in 3D fast spin echo. Magn Reson Med 2008;60:640-49 CrossRef Medline

19. Hennig J, Weigel M, Scheffler K. Multiecho sequences with variable refocusing flip angles: optimization of signal behavior using smooth transitions between pseudo steady states (TRAPS). Magn Reson Med 2003;49:527-35 CrossRef Medline

20. Busse RF, Hariharan $\mathrm{H}, \mathrm{Vu} \mathrm{A}$, et al. Fast spin-echo sequences with very long echo trains: design of variable refocusing flip angle schedules and generation of clinical T2 contrast. Magn Reson Med 2006;55:1030-37 CrossRef Medline

21. Brau AC, Beatty PJ, Skare S, et al. Comparison of reconstruction accuracy and efficiency among autocalibrating data-driven parallel imaging methods. Magn Reson Med 2008;59:382-95 CrossRef Medline

22. Pan J, Quon JL, Johnson E, et al. Rapid-sequence brain magnetic resonance imaging for Chiari I abnormality. J Neurosurg Pediatr 2018;22:158-64 CrossRef Medline

23. Brenner D, Elliston $\mathrm{C}$, Hall E, et al. Estimated risks of radiationinduced fatal cancer from pediatric CT. AJR Am J Roentgenol 2001;176:289-96 CrossRef Medline

24. Thompson EM, Baird LC, Selden NR. Results of a North American survey of rapid-sequence MRI utilization to evaluate cerebral ventricles in children. J Neurosurg Pediatr 2014;13:636-40 CrossRef Medline

25. Koral K, Blackburn T, Bailey AA, et al. Strengthening the argument for rapid brain MR imaging: estimation of reduction in lifetime attributable risk of developing fatal cancer in children with shunted hydrocephalus by instituting a rapid brain MR imaging protocol in lieu of head CT. AJNR Am J Neuroradiol 2012;33:1851-54 CrossRef Medline

26. Malviya S, Voepel-Lewis T, Prochaska G, et al. Prolonged recovery and delayed side effects of sedation for diagnostic imaging studies in children. Pediatrics 2000;105:E42 CrossRef Medline

27. Keerthivasan MB, Winegar B, Udayasankar U, et al. An optimized single-shot sequence for fast $\mathrm{T} 2 \mathrm{w}$ imaging of the brain. In: Proceedings of the Joint Annual Meeting International Society for Magnetic Resonance in Medicine and the European Society for Magnetic Resonance in Medicine and Biology, Paris, France. June $16-21,2018$ 